Open Systems \& Information Dynamics Vol. 24, No. 4 (2017) 1799001 (4 pages) DOI:10.1142/S1230161217990013

(C) World Scientific Publishing Company

\title{
Index of Vol. 24 (2017)
}

Accardi L., A. Boukas, And Y.-G. Lu, The Vacuum Distributions of the Truncated Virasoro Fields are Products of Gamma Distributions

1750004

AcCARdi L And Y. G. Lu, The First 40 Years of GKSL Generators and Some Proposal for the Future

1740002

Adesso G., see González J. O., L. A. Correa, G. Nocerino, J. P. Palao, D. Alonso, and G. Adesso

1740010

Ajisaka S., see Kimura G., S. Ajisaka, and K. Watanabe

1740009

Alicki R., From the GKLS Equation to the Theory of Solar and Fuel Cells

1740007

Alonso D., see González J. O., L. A. Correa, G. Nocerino, J. P. Palao, D. Alonso, and G. Adesso

1740010

Benatti F., R. Floreanini, F. Franchini, and U. Marzolino, Remarks on Entanglement and Identical Particles

1740004

Benatti F., S. Khabbazi Oskouei, and A. Shafiei Deh Abad, Quantum Entropy and Complexity

1750005

Borji M. A., see Mortezapour A., M. A. Borji, D.-K. Park, R. Lo Franco 1740006

Boukas A., see AcCArdi L., A. Boukas, And Y.-G. Lu 1750004

Buchleitner A., see Kropf C. M., V. N. Shatokhin, and A. Buchleitner 1740012

Burgarth D., P. Facchi, G. Garnero, H. Nakazato, S. Pascazio, and K. Yuasa, Can Decay Be Ascribed to Classical Noise?

1750001

Chruściński D. And S. PAscazio, A Brief History of the GKLS Equation

1740001

Ciaglia F. M., F. Di Cosmo, A. Ibort, M. Laudato, and G. Marmo, Dynamical Vector Fields on the Manifold of Quantum States

1740003

Ciccarello F., see lorenzo S., F. Ciccarello, G. M. Palma, and B. Vacchini

1740011

Correa L. A., see González J. O., L. A. Correa, G. Nocerino, J. P. Palao, D. Alonso, and G. Adesso

1740010

Di Cosmo F., see Ciaglia F. M., F. Di Cosmo, A. Ibort, M. Laudato, and G. MARMo

1740003

Diósi L. AND A. Tilloy, On GKLS Dynamics for Local Operations and Classical Communication

1740020

Dirr G., see Schulte-HerbrügGen T., G. Dirr, and R. Zeier 1740019

El Gheteb S., see Mukhamedov F. and S. El Gheteb 1750010

Facchi P., see Burgarth D., P. Facchi, G. Garnero, H. Nakazato, S. Pascazio, AND K. YUASA

FACChi P., M. Ligabò, AND K. YuASA, On the Derivation of the GKLS Equation for Weakly Coupled Systems

Fagnola F., E. SAsso and V. Umanità, Structure of Uniformly Continuous Quantum Markov Semigroups with Atomic Decoherence-free Subalgebra

1750001

1740017

1740005 
Author Index

Floreanini R., see Benatti F., R. Floreanini, F. Franchini, and U. Marzolino Franchini F., see Benatti F., R. Floreanini, F. Franchini, and U. Marzolino Furusho K., S. Iriyama, and M. Ohya, Chaos Amplification Process Can Be Described by the GKSL Master Equation

Garnero G., see Burgarth D., P. Facchi, G. Garnero, H. Nakazato, S. PasCAZIO, AND K. YUASA

GiRALDI F., Bath Energy for Correlated Initial States vs. Information Flow in Local Dephasing Channels

González J. O., L. A. Correa, G. Nocerino, J. P. Palao, D. Alonso, and G. Adesso, Testing the Validity of the 'Local' and 'Global' GKLS Master Equations on an Exactly Solvable Model

Hiesmayr B. C., The GKLS Master Equation in High Energy Physics

1750001

1750006

Holevo A. S., see Siemon I., A.S. Holevo, And R. F. Werner

1740010

1740008

1740015

Ibort A., see Ciaglia F. M., F. Di Cosmo, A. Ibort, M. Laudato, and G. Marmo Iriyama S., see Furusho K., S. Iriyama, AND M. OHyA

1740003

1750008

Kamizawa T., On Some Algebraic Procedure for Block-Diagonal Decompositions of Open Dynamical Systems

1750002

Khabbazi Oskouei S., see Benatti F., S. Khabbazi Oskouei, and A. Shafiei Deh ABAD

Kimura G., S. Ajisaka, And K. Watanabe, Universal Constraints on Relaxation Times for $d$-Level GKLS Master Equations

Kropf C. M., V. N. Shatokhin, and A. Buchleitner, Open System Model for Quantum Dynamical Maps with Classical Noise and Corresponding Master Equations

1750005

1740009

Laudato M., see Ciaglia F. M., F. Di Cosmo, A. Ibort, M. Laudato, and G. MARMO

Li Y. L., see XiaO X., J. J. Wu, W. J. Zhong, AND Y.L. Li

1740012

1740003

1750003

Ligabò M., see FACChi P., M. Ligabò, AND K. YuASA

1740017

Lo Franco R., see Mortezapour A., M. A. Borji, D.-K. Park, R. Lo Franco

1740006

Lorenzo S., F. Ciccarello, G.M. Palma, and B. Vacchini, Quantum NonMarkovian Piecewise Dynamics from Collision Models

1740011

Lu Y.-G., see Accardi L., A. Boukas, And Y.-G. LU

1750004

LU Y. G., see ACCARDi L AND Y. G. LU

1740002

Macchiavello C. And M. F. Sacchi, Detection of Properties and Capacities of Quantum Channels

Maciejewski A. J., see Musumbu D. P., M. Przybylska, and A. J. Maciejewski

MaćKowiak J., Bose-Einstein Condensation of a Stochastic Liquid

1750009

Maniscalco S., see Nokkala J., S. Maniscalco, and J. Pillo

1740018

Marmo G., see Ciaglia F. M., F. Di Cosmo, A. Ibort, M. Laudato, and G. Marmo

1740003

Marzolino U., see Benatti F., R. Floreanini, F. Franchini, and U. Marzolino

1740004 
Index of Vol. 24 (2017)

Megier N. And W. T. Strunz, Total State Dynamics in the GKSL Regime

Milz S., F. A. Pollock, And K. Modi, An Introduction to Operational Quantum Dynamics

Modi K., see Milz S., F. A. Pollock, And K. Modi

Mortezapour A., M. A. BorJi, D.-K. PArk, R. Lo Franco, Non-Markovianity and Coherence of a Moving Qubit inside a Leaky Cavity

Mukhamedov F. And S. El Gheteb, Uniqueness of Quantum Markov Chain Associated with XY-Ising Model on Cayley Tree of Order Two

Musumbu D. P., M. Przybylska, and A. J. Maciejewski, Probing the Eigenstates Thermalization Hypothesis with Many-Particle Quantum Walks on Lattices

Nakazato H., see Burgarth D., P. Facchi, G. Garnero, H. Nakazato, S. PasCAZIO, AND K. YUASA

Nocerino G., see González J. O., L. A. Correa, G. Nocerino, J. P. Palao, D. Alonso, and G. Adesso

Nokkala J., S. Maniscalco, and J. Pillo, Non-Markovianity over Ensemble Averages in Quantum Complex Networks

Palao J.P., see González J. O., L. A. Correa, G. Nocerino, J. P. Palao, D. Alonso, and G. Adesso

Palma G. M., see Lorenzo S., F. Ciccarello, G. M. Palma, and B. Vacchini

Pascazio S., see Burgarth D., P. Facchi, G. Garnero, H. Nakazato, S. Pascazio, AND K. YUASA

Pascazio S., see Chruściński D. and S. Pascazio

1750001

Piilo J., see Nokkala J., S. Maniscalco, and J. Pillo

Pollock F. A., see Milz S., F. A. Pollock, and K. Modi

Przybylska M., see Musumbu D. P., M. Przybylska, and A. J. Maciejewski

Schulte-Herbrüggen T., G. DirR, And R. Zeier, Quantum Systems Theory Viewed from Kossakowski-Lindblad Lie Semigroups - and Vice Versa

Shafiei Deh Abad A., see Benatti F., S. Khabbazi Oskouei, and A. Shafiei Deh ABAD

Shatokhin V. N., see Kropf C. M., V. N. Shatokhin, And A. Buchleitner

Siemon I., A.S. Holevo, And R. F. Werner, Unbounded Generators of Dynamical Semigroups 
Watanabe K, see Kimura G., S. Ajisaka, and K. Watanabe

Xiao X., J. J. Wu, W. J. Zhong, And Y.L. Li, Non-Markovian Dynamics of Spin Squeezing Under Detuning Modulation

1750003

Yuasa K., see Burgarth D., P. Facchi, G. Garnero, H. Nakazato, S. Pascazio, AND K. YUASA

1750001

YuAsa K., see Facchi P., M. Ligabò, And K. Yuasa

1740017

Zeier R., see Schulte-Herbrüggen T., G. Dirr, and R. Zeier

1740019

Zhong W. J., see Xiao X., J. J. Wu, W. J. Zhong, and Y. L. Li

1750003 